\title{
Sulla consistenza della determinazione dei numeri caratteristici dei flocculi di calcio
}

\author{
G. GODOLI \\ ricevuto il 3 novembre 1959
}

1. - E noto come nel congresso dell'Unione Astronomica Internazionale tenutosi a Leida nel 1928 venisse raccomandato di stimare l'attività globale dei flocculi e dei filamenti solari con i numeri caratteristici che, in una scala da 0 a 5 , dovevano rappresentare sinteticamente sia l'area che l'intensità dei fenomeni $\left({ }^{1,2}\right)$.

Abbiamo già parlato in questa sede delle vicissitudini che la pubblicazione di questi indici ha subito $\left({ }^{1}\right)$. Recentemente, in seguito a ricerche eseguite ali'Osservatorio di Arcetri $\left({ }^{1,3,4}\right)$, è stata riconosciuta l'utilità di continuare la pubblicazione di questi indici, anche per quei fenomeni per cui si effettuano misure più dettagliate. Si hanno infatti indizi che stime dell'attività globale con i numeri caratteristici e misure di area offrano informazioni diverse, almeno per quanto riguarda l'attività dei flocculi di calcio $\left({ }^{4}\right)$.

L'Osservatorio di Arcetri ha ripreso la pubblicazione dei numeri caratteristici dal 1950. Annualmente vengono pubblicati in questa sede i valori giornalieri e le medie mensili ed annuali dei numeri caratteristici dei flocculi d'idrogeno e di calcio e dei filamenti d'idrogeno determinati ad Arcetri $\left({ }^{5}\right)$. Per il periodo 1942-1949 sono state pubblicate, sempre in questa sede $\left(^{1}\right)$, solo le medie mensili dei numeri caratteristici dei tre fenomeni determinati ad Arcetri. Precedentemente, i valori giornalieri e le medie mensili, semestrali ed annuali dei numeri caratteristici dei tre fenomeni determinati ad Arcetri sono stati pubblicati, insieme alle determinazioni eseguite negli altri Osservatori, nel bollettino "Character figures of solar phenomena" che dal 1939 ha preso il nome di "Quarterly bulletin on solar activity ".

Dal 1948 anche l'Osservatorio di Tokio ha pubblicato fra l'altro nel suo "Bulletin of solar phenomena " $\mathrm{i}$ valori giornalieri e le medie mensili dei numeri caratteristici dei flocculi di calcio $\left(^{6}\right)$. 
Recentemente anche l'Osservatorio di Ikomasan, dell'Università di Kyoto, ha pubblicato un volume di osservazioni solari nel quale sono riportati fra l'altro i valori giornalieri e le medie mensili dei numeri caratteristici dei flocculi di calcio per il periodo Settembre 1946-Dicembre $1954\left({ }^{7}\right)$.

Avendo a disposizione questo materiale abbiamo creduto opportuno eseguire un confronto fra le determinazioni di numeri caratteristici dei flocculi di calcio effettuate ad Arcetri, ad Ikomasan e Tokio soprattutto allo scopo di valutarne la consistenza.

2. - Nella_Tabella I sono riportate le medie mensili, semestrali ed annuali dei numeri caratteristici determinati ad Arcetri, Ikomasan, Tokio. Le medie sono state calcolate per i giorni in cui la determinazione era stata effettuata sia ad Arcetri che ad Ikomasan oppure sia ad Arcetri che a Tokio. I numeri in corsivo sotto le medie indicano il numero di giorni di osservazione.

La Tabella è illustrata dai grafici di figg. 1-9. Nei grafici di figg. 1 e 2 sono riportati i confronti fra le medie mensili di Arcetri e quelle di Ikomasan e Tokio. Nel grafico di fig. 3 sono riportati l'andamento dei rapporti fra le medie mensili di Ikomasan e Tokio e quelle di Arcetri.

I grafici di figg. 4-6 e $7-9$ hanno analogo significato per le medie semestrali e quelle annuali.

3. - L'esame della Tabella e dei grafici permette.di trarre le seguenti conclusioni:

a) date le incertezze e le limitazioni inerenti al metodo di misura, la consistenza delle stime dei numeri caratteristici appare assai soddisfacente;

b) i numeri caratteristici determinati ad Arcetri hanno la tendenza ad essere maggiori di quelli determinati ad Ikomasan e Tokio. Dalle figg. 3,3 bis, 6,9 risulta che questa tendenza è più accentuata nei periodi di minor attività solare.

c) La notevole discrepanza del 1954 è tale solo percentualmente. In effetti si tratta, come risulta dalla tabella 1 , di divergenze non superiori a 0.2 . 
Tabella I - Medie mensili, Semestrali ed annuali dei Numeri caratteristici determinati ad Arcetri, Iromasan, Tokio. (I numeri in corsivo sotto le medie indicano il numero di giorni di osservazione).

\begin{tabular}{|c|c|c|c|c|c|c|c|c|c|c|c|c|c|c|c|c|}
\hline \multirow{3}{*}{ Anno } & \multirow{3}{*}{$\begin{array}{l}0 \\
0 \\
0 \\
0 \\
0 \\
0 \\
0 \\
0 \\
0 \\
0 \\
0\end{array}$} & \multicolumn{12}{|c|}{$\mathrm{mes} \theta$} & \multirow{2}{*}{\multicolumn{2}{|c|}{ Semestre }} & \multirow{3}{*}{ Anno } \\
\hline & & \multirow{2}{*}{ 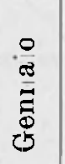 } & \multirow{2}{*}{$\begin{array}{l}\stackrel{0}{\pi} \\
\frac{1}{1} \\
0 \\
0 \\
0 \\
0=1\end{array}$} & \multirow[b]{2}{*}{ 莡 } & \multirow[b]{2}{*}{$\begin{array}{l}0 \\
\frac{D}{E} \\
\frac{\theta}{4}\end{array}$} & \multirow[b]{2}{*}{ 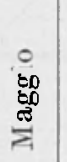 } & \multirow[b]{2}{*}{ 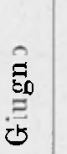 } & \multirow[b]{2}{*}{ 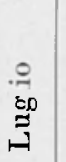 } & \multirow[b]{2}{*}{$\begin{array}{l}0 \\
i n \\
80 \\
80 \\
4\end{array}$} & \multirow{2}{*}{ 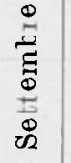 } & \multirow[b]{2}{*}{$\begin{array}{l}0 \\
\frac{1}{0} \\
0 \\
\frac{1}{0} \\
0\end{array}$} & \multirow{2}{*}{$\begin{array}{l}\vec{E} \\
\vec{E} \\
\vec{G} \\
\dot{D} \\
0 \\
z\end{array}$} & \multirow{2}{*}{$\begin{array}{c}0 \\
\frac{0}{3} \\
\frac{3}{3} \\
\frac{3}{0} \\
\frac{0}{0}\end{array}$} & & & \\
\hline & & & & & & & & & & & & & & I & II & \\
\hline 1950 & $\left\{\begin{array}{l}\mathrm{A} \\
\mathrm{I} \\
\mathrm{A} \\
\mathrm{T}\end{array}\right.$ & $\begin{array}{l}2.9 \\
2.7 \\
2 \\
2.7 \\
1.9 \\
4\end{array}$ & \begin{tabular}{|l|}
3.0 \\
2.8 \\
3 \\
2.9 \\
3.0 \\
2
\end{tabular} & \begin{tabular}{|l|}
2.6 \\
2.2 \\
5 \\
2.6 \\
2.2 \\
5
\end{tabular} & $\begin{array}{l}2.8 \\
2.3 \\
2 \\
2.8 \\
2.5 \\
2\end{array}$ & $\begin{array}{l}2.6 \\
2.4 \\
8 \\
2.5 \\
2.3 \\
9\end{array}$ & $\begin{array}{l}2.2 \\
2.6 \\
6 \\
2.4 \\
1.8 \\
4\end{array}$ & \begin{tabular}{|c|}
2.3 \\
2.3 \\
14 \\
2.3 \\
2.1 \\
$1 \%$
\end{tabular} & \begin{tabular}{|l|}
2.3 \\
2.3 \\
9 \\
2.3 \\
2.0 \\
5
\end{tabular} & $\begin{array}{l}1.7 \\
2.0 \\
3 \\
1.5 \\
1.5 \\
3\end{array}$ & $\begin{array}{l}1.9 \\
2.1 \\
5 \\
1.8 \\
1.7 \\
3\end{array}$ & \begin{tabular}{l|}
1.8 \\
1.5 \\
2 \\
2.1 \\
1.8 \\
2
\end{tabular} & $\begin{array}{l}- \\
- \\
- \\
-\end{array}$ & $\begin{array}{c}2.57 \\
2.47 \\
26 \\
2.58 \\
2.21 \\
26\end{array}$ & $\begin{array}{c}2.13 \\
2.19 \\
33 \\
2.14 \\
1.93 \\
30\end{array}$ & $\begin{array}{c}2.32 \\
2.31 \\
59 \\
2.34 \\
2.06 \\
56\end{array}$ \\
\hline 1951 & $\begin{array}{l}\left\{\begin{array}{l}\mathrm{A} \\
\mathrm{I}\end{array}\right. \\
\mathrm{A} \\
\mathrm{T}\end{array}$ & $\begin{array}{l}1.8 \\
1.7 \\
3 \\
1.6 \\
1.2 \\
5\end{array}$ & $\begin{array}{l}1.6 \\
1.6 \\
3 \\
2.2 \\
2.0 \\
1\end{array}$ & $\begin{array}{l}1.3 \\
1.3 \\
4 \\
0.9 \\
0.5 \\
1\end{array}$ & $\begin{array}{l}2.0 \\
1.4 \\
4 \\
2.1 \\
2.3 \\
2\end{array}$ & $\begin{array}{l}2.4 \\
2.4 \\
7 \\
2.3 \\
2.5 \\
7\end{array}$ & $\begin{array}{l}2.5 \\
2.0 \\
7 \\
2.6 \\
2.2 \\
7\end{array}$ & \begin{tabular}{|l|}
1.3 \\
1.4 \\
4 \\
1.8 \\
1.8 \\
5
\end{tabular} & $\mid \begin{array}{c}2.0 \\
1.5 \\
14 \\
1.9 \\
1.8 \\
9\end{array}$ & $\begin{array}{l}2.0 \\
1.9 \\
4 \\
1.8 \\
1.5 \\
1\end{array}$ & $\begin{array}{l}1.5 \\
1.2 \\
4 \\
1.6 \\
0.5 \\
1\end{array}$ & $\begin{array}{l}1.9 \\
1.2 \\
2 \\
2.0 \\
1.5 \\
3\end{array}$ & $\begin{array}{l}1.7 \\
1.2 \\
2 \\
1.8 \\
0.5 \\
2\end{array}$ & $\begin{array}{c}2.04 \\
1.82 \\
28 \\
2.17 \\
2.00 \\
23\end{array}$ & $\begin{array}{c}1.80 \\
1.44 \\
30 \\
1.87 \\
1.57 \\
21\end{array}$ & $\begin{array}{c}1.92 \\
1.63 \\
58 \\
2.02 \\
1.80 \\
44\end{array}$ \\
\hline 1952 & $\left\{\begin{array}{l}\mathrm{A} \\
\mathrm{I} \\
\mathrm{A} \\
\mathrm{T}\end{array}\right.$ & $\begin{array}{l}1.5 \\
1.7 \\
4 \\
1.5 \\
1.1 \\
7\end{array}$ & \begin{tabular}{|l|}
1.0 \\
0.8 \\
5 \\
0.8 \\
0.4 \\
4
\end{tabular} & \begin{tabular}{|l|}
0.6 \\
0.7 \\
4 \\
0.4 \\
0.5 \\
3
\end{tabular} & $\left|\begin{array}{l}0.8 \\
0.5 \\
3 \\
0.8 \\
0.5 \\
4\end{array}\right|$ & $\begin{array}{l}0.9 \\
0.6 \\
6 \\
0.9 \\
0.5 \\
3\end{array}$ & $\begin{array}{l}1.3 \\
0.8 \\
4 \\
0.6 \\
0.4 \\
4\end{array}$ & $\left|\begin{array}{l}0.7 \\
0.7 \\
9 \\
1.0 \\
0.9 \\
8\end{array}\right|$ & \begin{tabular}{|}
1.5 \\
1.0 \\
11 \\
1.4 \\
1.6 \\
5
\end{tabular} & $\begin{array}{l}1.7 \\
1.6 \\
6 \\
1.5 \\
1.5 \\
4\end{array}$ & $\begin{array}{l}0.5 \\
0.5 \\
4 \\
0.5 \\
0.6 \\
4\end{array}$ & $\begin{array}{l}1.0 \\
0.6 \\
1 \\
1.0 \\
1.0 \\
1\end{array}$ & $\begin{array}{l}1.3 \\
1.3 \\
2 \\
1.6 \\
2.0 \\
1\end{array}$ & $\begin{array}{c}1.00 \\
0.87 \\
26 \\
0.92 \\
0.64 \\
25\end{array}$ & $\begin{array}{l}1.15 \\
1.00 \\
33 \\
1.10 \\
1.17 \\
23\end{array}$ & $\begin{array}{c}1.08 \\
0.94 \\
\tilde{59} \\
1.01 \\
0.90 \\
48\end{array}$ \\
\hline 1953 & $\begin{array}{l}\left\{\begin{array}{l}\mathrm{A} \\
\mathrm{I}\end{array}\right. \\
\qquad \mathrm{A} \\
\mathrm{T}\end{array}$ & $\begin{array}{l}1.0 \\
0.7 \\
3 \\
1.2 \\
1.1 \\
5\end{array}$ & \begin{tabular}{|l|}
0.4 \\
0.5 \\
1 \\
0.1 \\
0.2 \\
3
\end{tabular} & $\begin{array}{c}0.2 \\
0.1 \\
9 \\
0.3 \\
0.1 \\
10\end{array}$ & $\begin{array}{l}0.4 \\
0.3 \\
5 \\
0.5 \\
0.5 \\
4\end{array}$ & $\begin{array}{l}0.7 \\
0.4 \\
5 \\
0.9 \\
0.4 \\
4\end{array}$ & $\begin{array}{l}1.4 \\
0.4 \\
2 \\
- \\
- \\
\end{array}$ & $\left|\begin{array}{l}0.5 \\
0.1 \\
7 \\
0.6 \\
0.3 \\
4\end{array}\right|$ & $\begin{array}{c}0.8 \\
0.4 \\
11 \\
1.4 \\
1.1 \\
4\end{array}$ & $\begin{array}{l}1.2 \\
0.5 \\
3 \\
1.4 \\
0.5 \\
1\end{array}$ & $\begin{array}{l}0.7 \\
0.3 \\
4 \\
1.1 \\
0.5 \\
2\end{array}$ & $\begin{array}{l}0.3 \\
0.1 \\
3 \\
0.2 \\
0.2 \\
7\end{array}$ & $\begin{array}{l}0.0 \\
0.1 \\
1 \\
0.0 \\
0.0 \\
2\end{array}$ & $\begin{array}{c}0.55 \\
0.29 \\
25 \\
0.55 \\
0.40 \\
26\end{array}$ & $\begin{array}{c}0.61 \\
0.27 \\
32 \\
0.64 \\
0.43 \\
20\end{array}$ & $\begin{array}{c}0.58 \\
0.28 \\
57 \\
0.59 \\
0.41 \\
46\end{array}$ \\
\hline 1954 & $\begin{array}{l}\left\{\begin{array}{c}\mathrm{A} \\
\mathrm{I}\end{array}\right. \\
\{\mathrm{A} \\
\mathrm{T}\end{array}$ & $\begin{array}{l}0.0 \\
0.0 \\
3 \\
0.0 \\
0.3 \\
2\end{array}$ & $\begin{array}{l}0.0 \\
0.0 \\
6 \\
0.0 \\
0.0 \\
5\end{array}$ & $\mid \begin{array}{l}0.1 \\
0.1 \\
4 \\
0.2 \\
0.3 \\
5\end{array}$ & $\begin{array}{l}0.3 \\
0.1 \\
4 \\
0.4 \\
0.5 \\
2\end{array}$ & $\begin{array}{l}0.0 \\
0.0 \\
2 \\
0.0 \\
0.0 \\
2\end{array}$ & $\begin{array}{l}0.0 \\
0.0 \\
2 \\
0.0 \\
0.0 \\
1\end{array}$ & \begin{tabular}{|l|}
0.2 \\
0.1 \\
7 \\
0.2 \\
0.3 \\
6
\end{tabular} & $\begin{array}{c}0.3 \\
0.1 \\
10 \\
0.3 \\
0.3 \\
9\end{array}$ & $\begin{array}{l}0.2 \\
0.1 \\
4 \\
0.2 \\
0.3 \\
4\end{array}$ & \begin{tabular}{l|}
0.3 \\
0.1 \\
3 \\
0.3 \\
0.5 \\
4
\end{tabular} & $\begin{array}{l}0.3 \\
0.2 \\
5 \\
0.3 \\
0.5 \\
4\end{array}$ & \begin{tabular}{|l|}
0.3 \\
0.1 \\
5 \\
0.3 \\
0.3 \\
5
\end{tabular} & $\begin{array}{c}0.06 \\
0.03 \\
21 \\
0.08 \\
0.18 \\
17\end{array}$ & $\begin{array}{c}0.26 \\
0.12 \\
34 \\
0.25 \\
11.34 \\
32\end{array}$ & $\begin{array}{c}0.19 \\
0.08 \\
55 \\
0.19 \\
0.29 \\
49\end{array}$ \\
\hline 1955 & $\left\{\begin{array}{l}\mathrm{A} \\
\mathrm{T}\end{array}\right.$ & $\begin{array}{l}0.7 \\
0.6 \\
4\end{array}$ & $\left|\begin{array}{l|}0.2 \\
0.3 \\
2\end{array}\right|$ & $\begin{array}{l}0.6 \\
0.5 \\
2\end{array}$ & $\begin{array}{c}0.8 \\
0.5 \\
10\end{array}$ & $\begin{array}{l}0.9 \\
0.6 \\
6\end{array}$ & $\begin{array}{l}1.7 \\
0.8 \\
3\end{array}$ & $\mid \begin{array}{c}1.2 \\
0.5 \\
13\end{array}$ & $\left|\begin{array}{l}2.0 \\
0.9 \\
8\end{array}\right|$ & $\begin{array}{l}1.2 \\
0.6 \\
5\end{array}$ & $\begin{array}{l}2.2 \\
1.0 \\
3\end{array}$ & \begin{tabular}{|l|}
3.0 \\
1.8 \\
6
\end{tabular} \mid & $\begin{array}{l}3.2 \\
1.9 \\
4\end{array}$ & $\begin{array}{c}0.86 \\
0.54 \\
27\end{array}$ & $\begin{array}{c}1.90 \\
0.99 \\
39\end{array}$ & $\begin{array}{c}1.47 \\
0.80 \\
66\end{array}$ \\
\hline 1956 & $\left\{\begin{array}{l}\mathrm{A} \\
\mathrm{T}\end{array}\right.$ & \begin{tabular}{l|}
2.8 \\
2.3 \\
3
\end{tabular} & $\begin{array}{l}2.7 \\
1.4 \\
7\end{array}$ & \begin{tabular}{|l|}
3.3 \\
2.2 \\
5
\end{tabular} & $\left|\begin{array}{l}3.7 \\
2.6 \\
5\end{array}\right|$ & $\begin{array}{l}4.0 \\
2.6 \\
\gamma\end{array}$ & $\begin{array}{l}4.0 \\
2.8 \\
4\end{array}$ & $\left|\begin{array}{l}3.5 \\
3.0 \\
6\end{array}\right|$ & $\begin{array}{l}4.0 \\
3.1 \\
6\end{array} \mid$ & $\begin{array}{l}3.5 \\
3.2 \\
6\end{array}$ & $\begin{array}{l}2.8 \\
2.6 \\
4\end{array}$ & $\begin{array}{l}3.8 \\
3.0 \\
2\end{array}$ & \begin{tabular}{|l|}
3.7 \\
3.0 \\
8
\end{tabular} & $\begin{array}{c}3.43 \\
2.27 \\
31\end{array}$ & $\begin{array}{l}3.56 \\
3.00 \\
32\end{array}$ & $\begin{array}{r}3.49 \\
2.74 \\
63\end{array}$ \\
\hline 1957 & $\left\{\begin{array}{l}\mathrm{A} \\
\mathrm{T}\end{array}\right.$ & $\begin{array}{r}3.7 \\
2.9 \\
10\end{array}$ & $\begin{array}{l}2.8 \\
2.0 \\
2\end{array}$ & $\begin{array}{c}3.6 \\
3.0 \\
11\end{array}$ & $\begin{array}{l}3.9 \\
3.3 \\
2\end{array}$ & $\begin{array}{l}3.6 \\
3.4 \\
8\end{array}$ & $\begin{array}{l}3.9 \\
3.5 \\
7\end{array}$ & $\begin{array}{l}3.4 \\
3.4 \\
7\end{array}$ & $\begin{array}{r}2.9 \\
3.0 \\
11\end{array}$ & $\begin{array}{l}3.5 \\
3.4 \\
6\end{array}$ & $\begin{array}{r}3.7 \\
3.9 \\
14\end{array}$ & $\begin{array}{l}3.5 \\
3.5 \\
6\end{array}$ & $\begin{array}{l}3.1 \\
3.4 \\
7\end{array}$ & $\begin{array}{l}3.64 \\
3.11 \\
40\end{array}$ & $\begin{array}{r}3.36 \\
3.46 \\
51\end{array}$ & $\begin{array}{r}3.48 \\
3.31 \\
91\end{array}$ \\
\hline
\end{tabular}




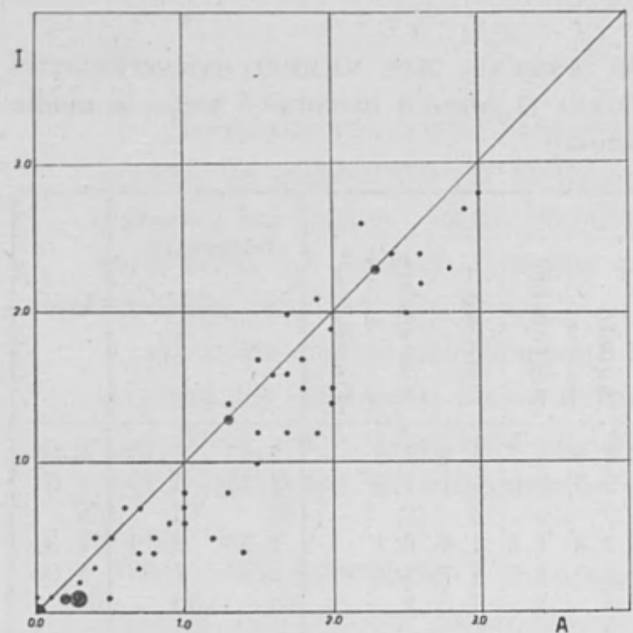

Fig. 1 - Confronto fra le medie mensili di Arcetri e quelle di Ikomasan.

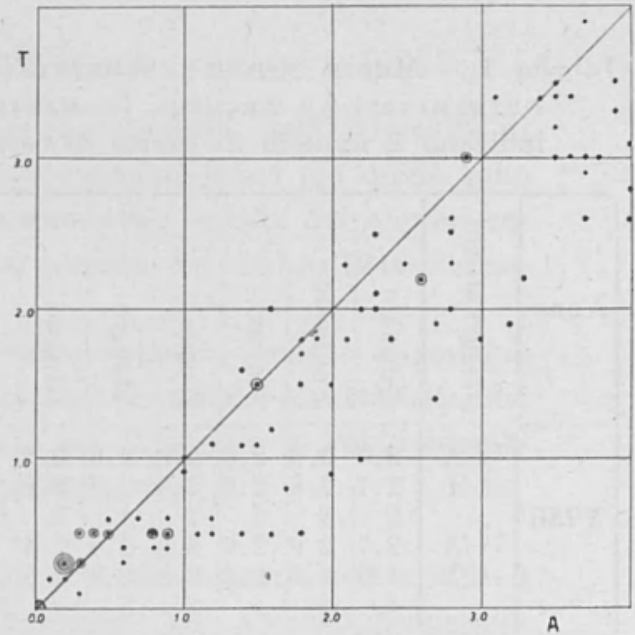

Fig. 2 - Confronto fra le medie mensili di Arcetri e quelle di Tokio.

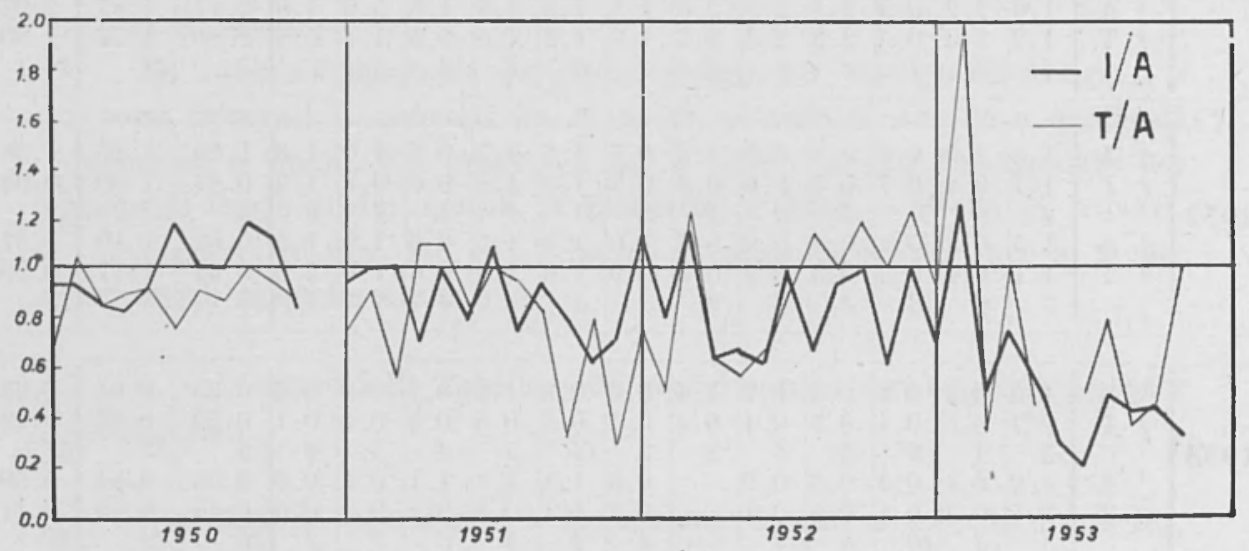

Fig. 3 - Andamento dei rapporti fra le medie mensili di Ikomasan e Tokio e quelle di Arcetri (1950-1953).

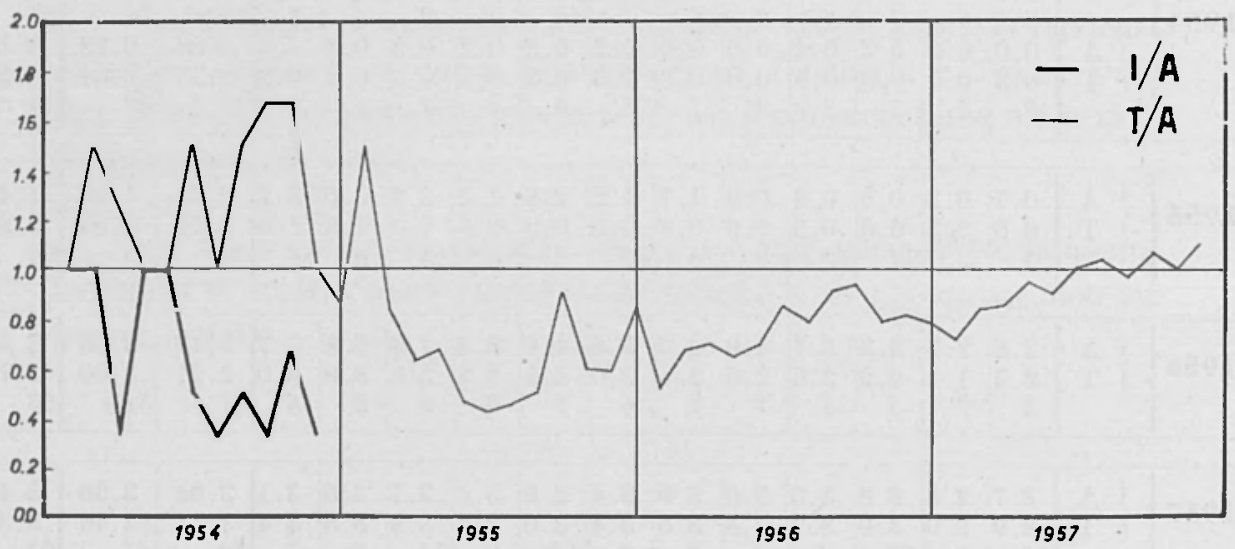

Fig. 3 bis - Andamento dei rapporti fra le medie mensili di Ikomasan e Tokio e quelle di Arcetri (1954-1957). 


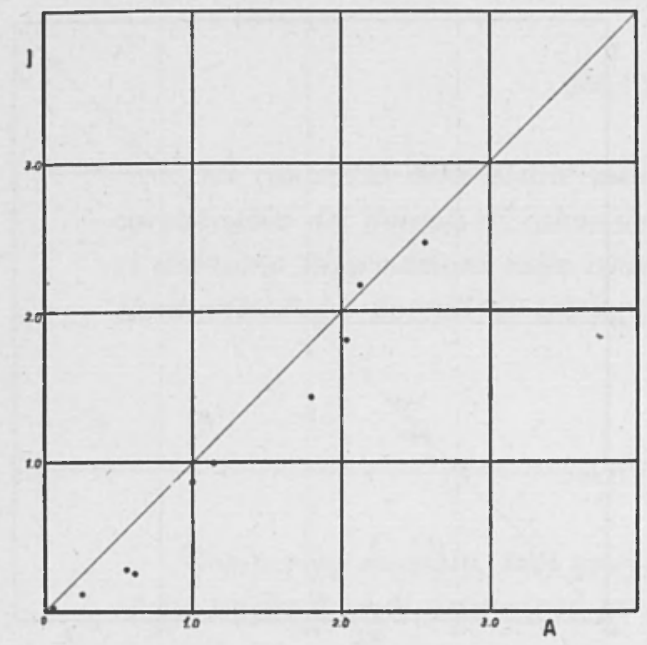

Fig. 4-Confronto fra le medie semestrali di Arcetri e quelle di Ikomasan.

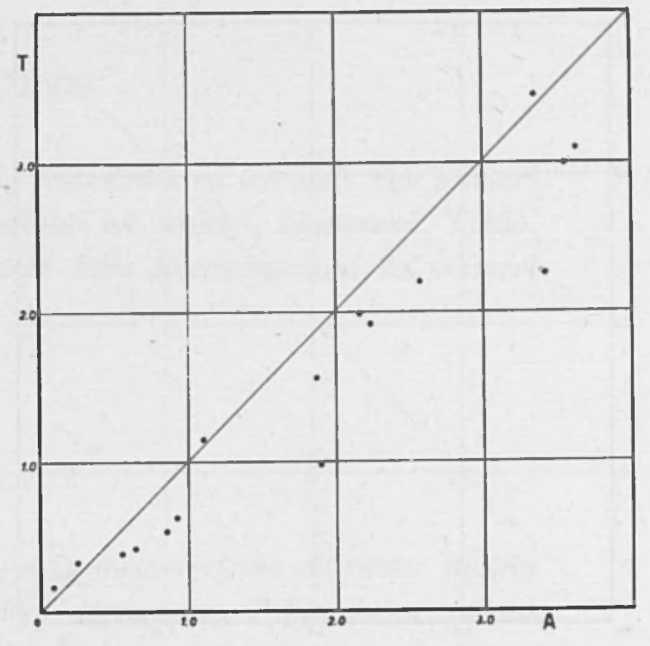

Fig. 5- Confronto fra le medie semestrali di Arcetri e quelle di Tokio.

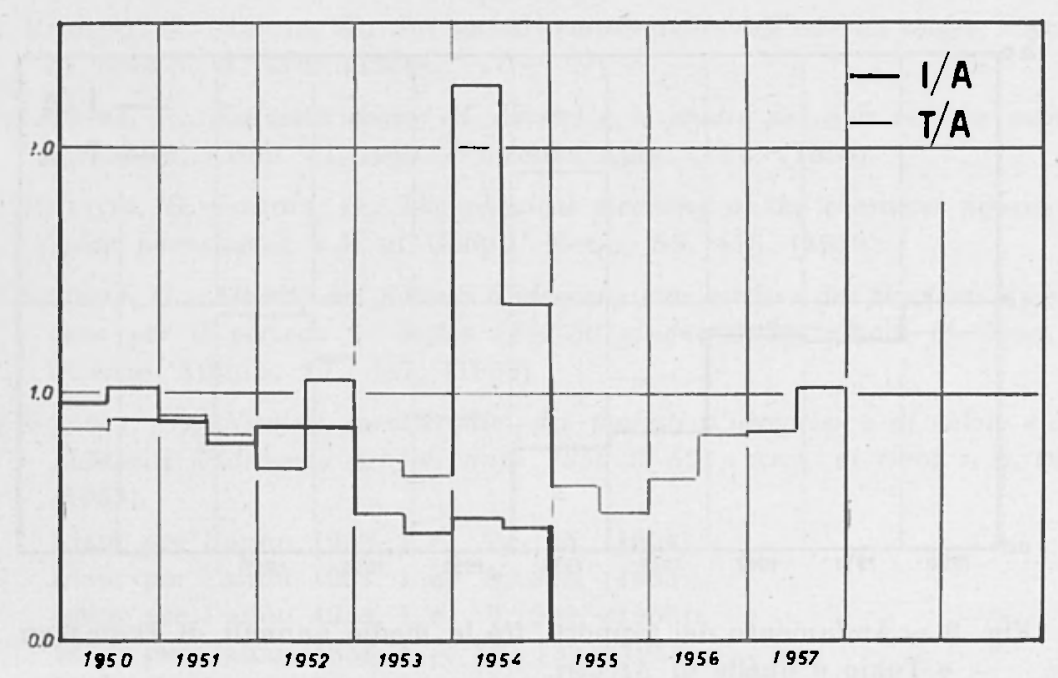

Fig. 6 - Andamento dei rapporti fra le medie semestrali di Ikomasan e Tokio e quelle di Arcetri. 


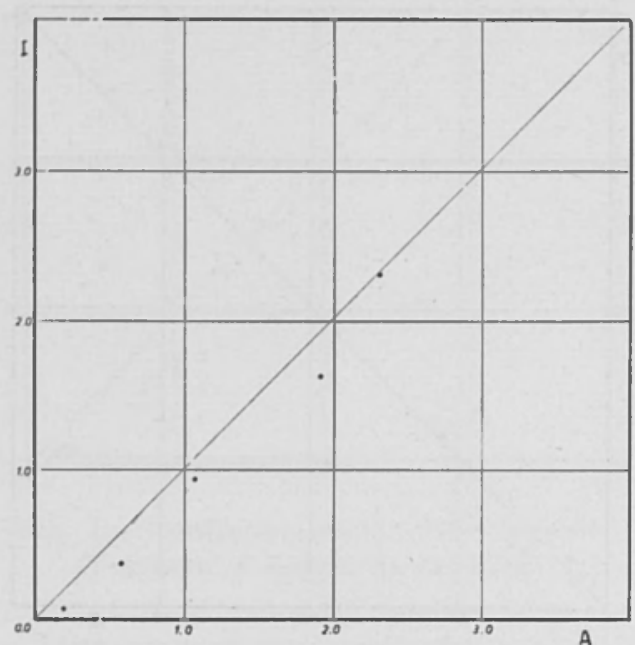

|Fig. 7 - Confronto fra le medie annuali di Arcetri e quelle di Ikomasan.

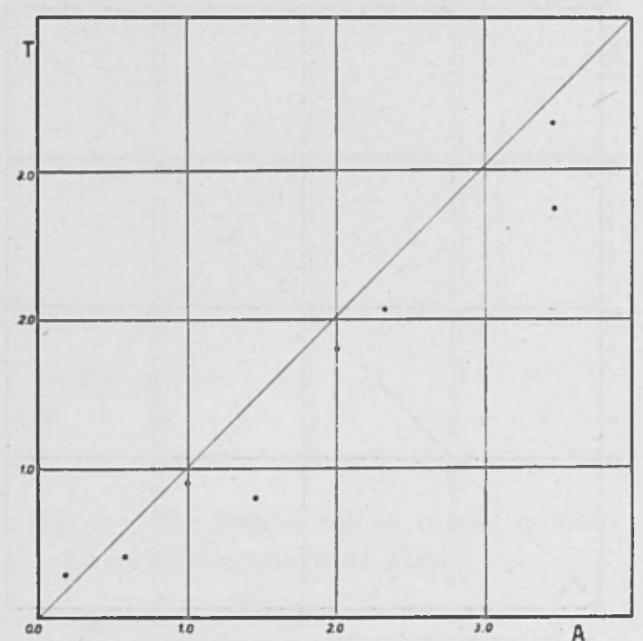

Fig. 8 - Confronto fra le medie annuali di Arcetri e quelle di Tokio.

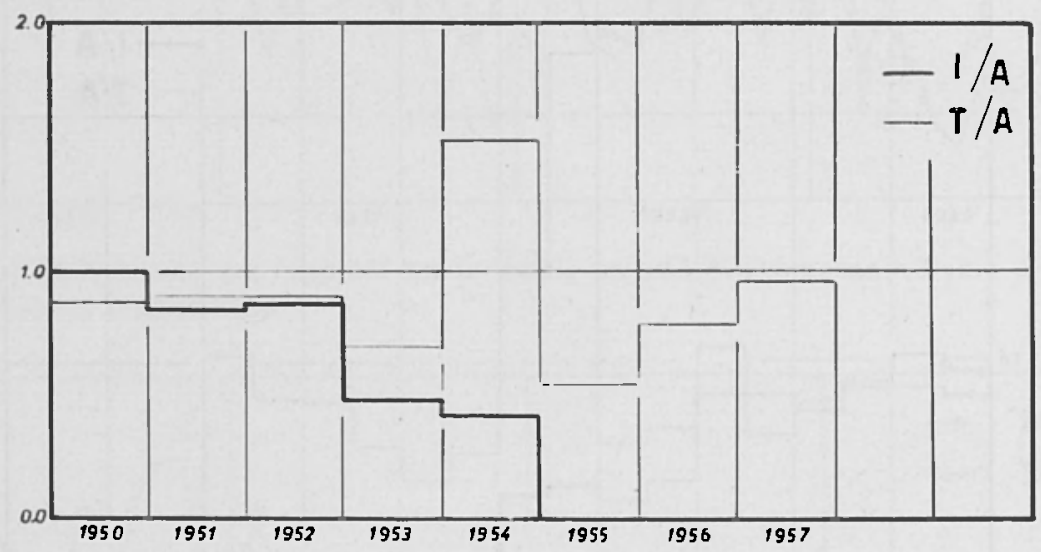

Fig. 9 - Andamento dei rapporti fra le medie annuali di Ikomasan e Tokio e quelle di Arcetri. 


\section{RIASSUNTO}

Dal confronto delle medie mensili, semestrali ed annuali dei numeri caratteristici dei flocculi di calcio determinati ad Arcetri, Ikomasan, Tokio, si deducono informazioni sulla consistenza delle determinazioni dei numeri caratteristici dei flocculi di calcio.

\section{$A B S T R A C T$}

Comparing monthly, half yearly, yearly means of the character figures of Ca bright flocculi determined at Arcetri, Ikomasan, Tokio, informations are deduced on the accuracy of the determinations of the character figures of Ca bright flocculi.

\section{BIBLIOGRAFIA}

(1) Righini, G.-Godol, G., Sui numeri caratteristici dell'attività solare, "Ann. di Geof. ", 3, 501, (1950).

(2) Godoli, G., La torre solare di Arcetri e lo studio del Sole in luce monocromatica, "Boll. di Geod. e Scienze Affini ", 14, (1955).

$\left.{ }^{3}\right)$ Righini, G.-Godoli, G., The physical meaning of the character figures of solar phenomena, "J. of Geoph. Res." 55, 415, (1950).

$\left({ }^{4}\right)$ Godoli, G., Attività dei flocculi d'idrogeno e di calcio e dei filamenti d'idrogeno per il periodo $1^{\text {o }}$ luglio 1957-30 giugno 1958, "Boll. di Geod. e Scienze Affini ", 17, 487, (1958).

(5) Godolı, G., Numeri caratteristici dei floceuli d'idrogeno e di calcio e dei filamenti d'idrogeno per gli anni 1950-51-52, "Ann. di Geof. ", 6. 199, (1953).

Idem per l'anno 1953, l. c. 7, 135, (1954).

Idem per l'anno 1954, l. c. 8, 325, (1955).

Idem per l'anno 1955, l. c. 9, 299, (1956).

Idem per l'anno 1956, l. c. 10, 133, (1957).

Idem per l'anno 1957, l. c. 10, 235, (1957).

Idem per l'anno 1958, 1. c. 11, 197, (1959).

(6) Tokio astr. obs. Bulletin of solar phenomena.

(7) Ikomasan solar observatory. Publ. 1 (1956). 